\title{
Echocardiographic Evaluation of Right Heart in Patients of Chronic Obstructive Pulmonary Disease
}

\author{
Pukhraj Singh Jeji1 ${ }^{1}$, Savita Kapila² ${ }^{2}$ Sunita Gupta ${ }^{3}$, Saurav Mittal ${ }^{4}$ \\ ${ }^{1}$ Department of Medicine, MMIMSR, MM(D)U, Mullana, Ambala, Haryana, India. ${ }^{2}$ Department of Medicine, MMIMSR, \\ MM(D)U, Mullana, Ambala, Haryana, India. ${ }^{3}$ Department of Medicine, MMIMSR, MM(D)U, Mullana, Ambala, Haryana, \\ India. ${ }^{4}$ Department of Medicine, MMIMSR, MM(D)U, Mullana, Ambala, Haryana, India.
}

\section{ABSTRACT}

\section{BACKGROUND}

Cardiac involvement in Chronic Obstructive Pulmonary Disease (COPD) is a significant cause of morbidity, mortality, and even death. We aimed to study the echocardiographic evaluation, especially of the right heart, in patients of COPD and to co-relate findings of echocardiography with the clinical profile and severity of COPD of such patients.

\section{METHODS}

We performed a cross-sectional study for a period of 2 years. The patients were subjected to necessary investigations and transthoracic echocardiography for evaluating the right heart parameters. Data was entered in MS Excel spreadsheet and analysis was done using Statistical Package for Social Sciences (SPSS) version 21.0. A p value of $<0.05$ was considered statistically significant.

\section{RESULTS}

Of the 50 COPD cases studied, the majority (22) fulfilled GOLD stage IV criteria followed by 14 cases in GOLD stage II. Right atrial pressure was increased in 52\% study subjects. Pulmonary artery systolic pressure had values of more than 25 mmHg in $68 \%$ of patients. RV thickness was $>5 \mathrm{~mm}$ in $56 \%$ subjects. TAPSE was measured to be $<17 \mathrm{~mm}$ in $50 \%$ COPD cases. A significant association was seen between severity of COPD and RA pressure, RV size, RV pressure/pulmonary artery pressure, RV thickness and TAPSE.

\section{CONCLUSIONS}

Significant prevalence of right heart dysfunction is found in COPD and there was a significant association of cardiac parameters with severity of COPD. Thus it is recommended that all patients of COPD should undergo right heart evaluation by echocardiography so that treatment modalities can be changed to minimize morbidity and mortality.

\section{KEY WORDS}

Chronic Obstructive Pulmonary Disease, Echocardiography, Ejection Fraction, Pulmonary Hypertension, Right Ventricle Outflow
Corresponding Author:

Pukhraj Singh Jeji,

H. No. 2157, Phase 7,

Sector 61, Mohali-160062,

Punjab, India.

E-mail: pukhrajsingh93@gmail.com

DOI: $10.14260 /$ jemds $/ 2020 / 210$

Financial or Other Competing Interests: None.

How to Cite This Article:

Jeji PS, Kapila S, Gupta $S$, et al. Echocardiographic evaluation of right heart in patients of chronic obstructive pulmonary disease. J. Evolution Med. Dent. Sci. 2020;9(12):974-979, DOI: $10.14260 /$ jemds/2020/210

Submission 23-01-2020, Peer Review 07-03-2020, Acceptance 12-03-2020, Published 23-03-2020.

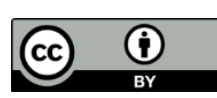




\section{BACKGROUND}

Chronic Obstructive Pulmonary Disease (COPD) is defined as a heterogenous group of diseases that have a common feature- narrowed airways which can cause the inability to exhale out completely. It is defined by GOLD as a preventable and treatable disease with some significant extrapulmonary effects. It is commonly encountered entity in the clinical practice. Although the most common cause of COPD is tobacco smoke, there are several other factors that can cause or make COPD worse, including environmental exposures and genetic (inherited) risk. Even the increasing pollution levels in our country becomes a major factor for the rise in COPD cases. As per medical research council report, it is expected to change from its status in 2000 as 4 th and 12 th most frequent cause of morbidity and mortality, to the 5th and 3rd leading cause of morbidity and mortality, respectively, in the year 2020.[1]

COPD is associated with significant extrapulmonary (systemic) effects among which cardiac manifestations are most common. The primary cardiovascular components affected in COPD patients include affect to pulmonary blood vessels, right ventricle (RV), and left ventricle leading to development of pulmonary hypertension (PAH), corpulmonale, right ventricular dysfunction, left ventricular dysfunction, and right ventricular dysfunction.[1] The extent of impairment of pulmonary action is important to be assessed to see the long-term prognosis.[1] The rise in the Pulmonary artery pressure has been found to be negatively correlated with the prognosis of COPD patients. ${ }^{[2-4]}$

Due to the systemic especially cardiac involvement in COPD, the mortality rates have significantly increased. Cardiovascular disease alone accounts for approximately $50 \%$ of all hospitalization and nearly one third of all deaths, if forced expiratory volume in one second (FEV1) $>50 \%$ of predicted. In more advanced disease cardiovascular disease account for $20 \%-25 \%$ of all deaths in COPD. ${ }^{[2-4]}$ The link between cardiovascular disease and COPD can be explained by many risk factors, including cigarette smoking, systemic effect of the inflammation, impaired vascular function, and hyperinflation of the lungs.[1] This link warrants the need to screen every patient of COPD for any cardiac manifestations.

Thus, the early and rapid non-invasive diagnosis of cardiac involvement and derangements in COPD patients holds the key as it may help better manage such patients. ECG has been long used for diagnosing RV hypertrophy. Though the detection of hypertrophy of RV in ECG has very high specificity but it has a disadvantage of low sensitivity. [2] In contrast, Echocardiography provides a rapid, non-invasive portable and accurate method to evaluate the right ventricle function, right ventricular filling pressure, tricuspid regurgitation, left ventricular function and valvular function. Two-dimensional echo has revolutionized the non-invasive evaluation of cases of COPD as well as prognosis and response to the treatment.[3] Studies have confirmed that echocardiographically derived estimates of pulmonary arterial pressure co-relate closely with pressures measured by right heart catheter.[1-4]

Thus, the present study was undertaken to assess the cardiac changes secondary to COPD by echocardiography, and to find out the correlation between echocardiographic findings and the severity of COPD using GOLD guidelines.

\section{METHODS}

A cross-sectional study was conducted at Ambala over a period of 2 years from September 2017 to September 2019. After Ethical Committee clearance, patients from the OPD and those admitted in Department of Medicine were selected after considering specific inclusion and exclusion criteria.

\section{Inclusion Criteria}

All patients of COPD coming to OPD or from indoor wards of Department of Medicine at MMIMSR, Mullana, as well as in ICU setting in the same institute. COPD is diagnosed as per guidelines of Global Initiative for Chronic Obstructive Lung Disease (GOLD), including clinical picture, X-Ray findings and Pulmonary function tests.

\section{Exclusion Criteria}

- Valvular heart disease and cardiomyopathy.

- Congenital heart disease.

- Systemic hypertension.

- Coronary artery disease.

- Chronic diseases like diabetes mellitus, renal failure, liver disorders, known cases of hypo or hyperthyroidism, collagen diseases and pregnancy.

- Patients in which right heart evaluation is not possible by echocardiography due to obscurity by hyper inflated lungs.

- Patients refusing consent.

- Patients with ankylosing spondylitis or other spinal deformities which can obstruct lung field.

All selected patients were asked in detail about their present, past, family and personal history especially about smoking, "choolah" use, and history of bronchial asthma. All necessary investigations and transthoracic echocardiography were done in all the patients. Right heart parameters were also studied. Tricuspid regurgitant jet and maximum jet velocity was also measured. Right ventricular systolic pressure was estimated based on modified Bernoulli's equation and was considered equal to the systolic pulmonary artery pressure (sPAP) in absence of right ventricular outflow tract obstruction. RAP was estimated on the basis of standard variation in size of inferior vena cava with inspiration. Tricuspid Annular Plane Systolic Excursion (TAPSE) was measured in M-mode and taken as a marker for RV dysfunction.

\section{Statistical Analysis}

Categorical variables were presented in number and percentage (\%) and continuous variables were presented as mean \pm SD and median. Normality of data was tested by Kolmogorov-Smirnov test. If the normality was rejected then non parametric test was used. Quantitative variables were compared using Independent $\mathrm{T}$ test/Mann-Whitney Test (when the data sets were not normally distributed) between the two groups. Qualitative variables were correlated using Chi-Square test. The data was entered in MS EXCEL spreadsheet and analysis was done using Statistical Package for Social Sciences (SPSS) version 21.0. A p value of $<0.05$ was considered statistically significant. 


\section{RESULTS}

Mean age ( \pm SD) of the patients in our study was $60.64 \pm$ 10.94 years. Majority (32\%) of the patients belonged to 61-70 years of age group followed by 51-60 years (28\%). Male predominance was present $(86 \%, n=43)$. Descriptive statistics of patients are given in Table 1. Right heart study distribution of study subjects is shown in Table 2 . Mean value of ejection fraction of study subjects was $57.94 \pm 5.53 \%$ and fractional shortening was $28.74 \pm 3.53 \%$. Of the 50 COPD cases that were studied, 1 case was GOLD stage I, 14 came in GOLD stage II, 13 cases fit into GOLD stage III and 22 cases fulfilled GOLD stage IV criteria. Twenty-three cases had dilated RV with maximum cases (15 of 22) being in GOLD stage IV category. Majority (68\%) of the patients had raised calculated right ventricular pressure with mean value of $37.04 \pm 16.48 \mathrm{mmHg}$, thus indicating pulmonary artery hypertension (PAH).

\begin{tabular}{|c|c|c|c|}
\hline Parameters & Mean \pm S.D. & \multicolumn{2}{|c|}{ Median (IQR) } \\
\hline Systolic blood pressure (mmHg) & $122.84 \pm 7.39$ & \multicolumn{2}{|c|}{$122(120-130)$} \\
\hline Diastolic blood pressure $(\mathrm{mmHg})$ & $77.12 \pm 5.64$ & \multicolumn{2}{|c|}{$80(70-80)$} \\
\hline Haemoglobin $(\mathrm{gm} / \mathrm{dl})$ & $12.42 \pm 2.23$ & \multicolumn{2}{|c|}{$12.4(10.500-14)$} \\
\hline TLC (/cumm) & $10914.4 \pm 3340.91$ & \multicolumn{2}{|c|}{$10700(8300-13500)$} \\
\hline Urea (mg) & $39.62 \pm 20.61$ & \multicolumn{2}{|c|}{$37.5(24-49)$} \\
\hline Serum sodium (mEq/L) & $137.88 \pm 4.79$ & \multicolumn{2}{|c|}{$138(135-141)$} \\
\hline Serum potassium $(\mathrm{mEq} / \mathrm{L})$ & $4.18 \pm 0.55$ & \multicolumn{2}{|c|}{$4.3(3.7-4.5)$} \\
\hline Platelets (/cumm) & $223160 \pm 74570.16$ & 212500 & $172000-276000)$ \\
\hline Chloride (mEq/L) & $99.2 \pm 4.98$ & & $0(97-102)$ \\
\hline Creatinine (mg/dl) & $1.11 \pm 0.33$ & 1.1( & $0.820-1.300)$ \\
\hline Echocar & diographic Paramet & ers & \\
\hline Aortic root $(\mathrm{mm})$ & $27.88 \pm 4.83$ & & $9(23-31)$ \\
\hline Left atrium (mm) & $34.06 \pm 5.7$ & & $1.5(30-38)$ \\
\hline RVID (D) (mm) & $34.84 \pm 7.99$ & & $4(28-42)$ \\
\hline IVST (D) (mm) & $11.63 \pm 2.84$ & & $1(10-13)$ \\
\hline IVST (S) (mm) & $14 \pm 2.35$ & & $4(12-15)$ \\
\hline LVID (D) (mm) & $43.53 \pm 8.04$ & & $4(39-50)$ \\
\hline LVID (S) (mm) & $29.66 \pm 6.6$ & & $0(25-33)$ \\
\hline LVPW (D) (mm) & $11.65 \pm 2.73$ & & $1(10-12)$ \\
\hline LVPW (S) (mm) & $14.24 \pm 2.67$ & & $4(12-16)$ \\
\hline & ppler Parameters & & \\
\hline E & $66.04 \pm 16.78$ & & $55-78.500)$ \\
\hline A & $81.48 \pm 15.72$ & & $1.5(72-92)$ \\
\hline $\mathrm{E} / \mathrm{A}$ & $0.83 \pm 0.25$ & 0.77 & $0.671-0.941)$ \\
\hline Table 1. Descriptiv & ve Parameters of & tudy Su & jects \\
\hline Parameter & Fre & quency & Percentage \\
\hline Right atrial Dimensio & & & \\
\hline Right at & trial pressure $(\mathrm{mmr}$ & & \\
\hline Normal & & 24 & $48.00 \%$ \\
\hline Abnormal (>8 mmHg) & & 26 & $52.00 \%$ \\
\hline Mean \pm S.D. & & & 4.29 \\
\hline Median(IQR) & & $10(5$ & $-15)$ \\
\hline Rigl & ht atrial size $(\mathrm{mm})$ & & \\
\hline Normal & & 27 & $54.00 \%$ \\
\hline Abnormal (>31 mm) & & 23 & $46.00 \%$ \\
\hline Mean \pm S.D. & & 32.44 & \pm 7.03 \\
\hline Median(IQR) & & $30(2$ & - 39) \\
\hline Right v & ventricular dimensi & & \\
\hline Right ventricular size (n & mm) & & \\
\hline Normal & & 27 & $54.00 \%$ \\
\hline Abnormal $(>40 \mathrm{~mm})$ & & 23 & $46.00 \%$ \\
\hline Mean \pm S.D. & & 34.84 & \pm 7.99 \\
\hline Median(IQR) & & $34(2$ & $3-42)$ \\
\hline Right ven & tricular thickness ( & mm) & \\
\hline Normal & & 22 & $44.00 \%$ \\
\hline Abnormal $(>5 \mathrm{~mm})$ & & 28 & $56.00 \%$ \\
\hline Mean \pm S.D. & & 7.16 & +2.92 \\
\hline Median(IQR) & & 7.5( & $-10)$ \\
\hline IVC an & nd pulmonary arter & & \\
\hline IVC inspiratory (mm) & & & \\
\hline Mean \pm S.D. & & 16.1 & \pm 5.11 \\
\hline Median(IQR) & & $16(1$ & - 21) \\
\hline Pulmor & nary artery size (mI & & \\
\hline Normal & & 21 & $42.00 \%$ \\
\hline Abnormal $(>20 \mathrm{~mm})$ & & 29 & $58.00 \%$ \\
\hline Mean \pm S.D. & & 19. & \pm 3.7 \\
\hline Median(IQR) & & $19(1$ & -21) \\
\hline & IVC collapse & & \\
\hline$<25 \%$ & & 9 & $18.00 \%$ \\
\hline$<50 \%$ & & 17 & $34.00 \%$ \\
\hline$>50 \%$ & & 24 & $48.00 \%$ \\
\hline Table 2. Right Heart & udy Distribution & of Study & Subjects \\
\hline
\end{tabular}

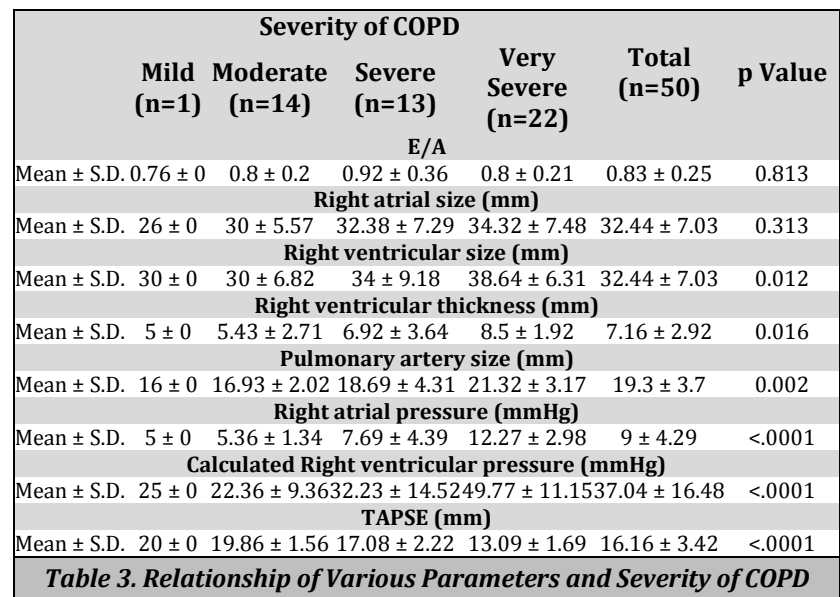

Half of the patients had normal TAPSE with mean value of $16.16 \pm 3.42 \mathrm{~mm}$. Right atrial pressure was increased in 26 out of 50 study subjects with mean being an elevated $9 \pm 4.29$ mm. Pulmonary artery systolic pressure was measured and 34 out of 50 cases had values $>25 \mathrm{mmHg}$ with mean being $37.04 \pm 16.48 \mathrm{mmHg}$. Out of these 34 cases having PAH, 7 were in GOLD stage II, 11 in stage II and 19 in stage IV. RV thickness was $>5 \mathrm{~mm}$ in 28 subjects out of 50 indicating RV hypertrophy. Of the 28 cases with RV hypertrophy, 23 were in GOLD stage III or IV. TAPSE was measured to be $<17 \mathrm{~mm}$ in 25 of 50 COPD cases indicative of RV dysfunction. TAPSE was $<17 \mathrm{~mm}$ in 21 out of 22 cases in very severe COPD category. Significant association was seen between right ventricular size and severity of COPD. Majority of the patients with mild, moderate and severe COPD had normal right ventricular size and on the other hand $68.18 \%$ of the patients with very severe COPD had abnormal right ventricular size. Statistically significant association was seen with severity of COPD and RA pressure, RV size, RV pressure/pulmonary artery pressure, RV thickness and TAPSE. Association of various parameters with severity of COPD is shown in Table 3.

\section{DISCUSSION}

The increasing incidence of COPD worldwide and in our country due to increasing smoking habits and pollution warrants the need to monitor and evaluate factors associated with increased and mortality and morbidity. The cardiac manifestations of COPD are numerous. Among them, the impairment of right ventricular dysfunction and pulmonary blood vessels are well known to complicate the clinical course of COPD and co-relate inversely with survival.

We found out that of the 50 COPD cases studied, the majority (22) fulfilled GOLD stage IV criteria followed by 14 cases in GOLD stage II. Right atrial pressure was increased in $52 \%$ study subjects. In our study, mean age of the patients was $60.64 \pm 10$ years. PAH is a common manifestation of COPD with its prevalence increasing with increasing duration of disease in COPD patients. These findings were concluded by Mutlu et al., Kessler et al., and Weitzenblum et al.[4,5,6] $\mathrm{A}$ higher PAP in older cases is in accordance in our study wherein the mean age of our study is $60.64 \pm 10.94$ years. The higher PAP in older age is explained by long duration of the disease and its more progression with advancing age. 
Weitzenblum et al. showed that one of the main characteristic of PAH in COPD cases is its mild-to-moderate degree, with a resting PAP in a stable state of disease ranging between 25 and 40 indicating a mild level of PAH.[7] In our study, PAH was present in $68 \%$ cases with a mean value of $37.04 \pm 16.48$, also indicating a mild level of PAH. In a trial by Scharf et al., only $5 \%$ patients had a PAP of $>35 \mathrm{mmHg}{ }^{[8]}$ Another study done by Chaouat A et al. showed only $2.7 \%$ COPD patients having severe PAH.[9] Similar findings were noted in a study by Abraham et al., Mohammed et al., and Jatav et al.[10,11,12] Thus, a resting PAP of $>35$ to $40 \mathrm{mmHg}$ is unusual in COPD patients. In our study, mean values of PAP varied greatly with severity of COPD. These variations can be attributed to the fact that most cases in our study were admitted in hospital having acute exacerbations of COPD and hence the raised PAP values can be easily explained.

In our study, among 2D echocardiographic variables, only mean value of right ventricular internal diameter (diastole) was higher, i.e. $34.84 \pm 7.99 \mathrm{~mm}$. Mean ejection fraction was $57.94 \pm 5.53$ percent, and none of the patients had any significant systolic LV dysfunction. The results are comparable with those of Moustapha et al. and Schena et al.[13,14] The results of our study also showed that none of the patients of COPD had evidence of any significant systolic dysfunction. Similar findings were reported by Mutlu et al.[4] Even in the pre-doppler era, studies have shown RV cavity dimensions being studied for variations with COPD. Trivedi HS et al., concluded that COPD patients have a tendency for right ventricular cavities being larger than standard values. In our study too, we have a significant correlation of RV size with COPD severity with 23 of 50 cases having dilated RV and mean value being $34.84 \pm 7.99 \mathrm{~mm} .{ }^{[15]}$ However, Mutlu et al. and Mohammed et al. did not find any significant correlation of RV size with COPD severity.[4,11]

It has been proven previously that RV thickness of $>6 \mathrm{~mm}$ is associated with $\mathrm{RV}$ pressure overload in patients with COPD.[16] Guidelines later established that RV thickness of $>5$ $\mathrm{mm}$ indicated RV hypertrophy and is the commonest right heart derangement seen in COPD patients and is indicative of cor-pulmonale.[17] In a study conducted on 34 patients, a significant correlation was established between severity of COPD patients by Danchin $\mathrm{N}$ et al.[18] In our study, mean RV thickness was $6.18 \pm 2.38$. There was significant correlation between RV thickness and severity of COPD. Mohammed et al. also reported similar finding.[11] In present study, RV hypertrophy is present in a majority of COPD cases taken in present study with mean value of RV thickness being $7.16 \pm$ $2.92 \mathrm{~mm} .{ }^{[18]}$ Our study showed significant correlation of PAP with FEV1. The number of patients of PAH also increased as the severity of disease increased by GOLD staging. Higham et al. showed that PAH is common in severe COPD and transtricuspid pressure gradient (TTPG) correlated with spirometric findings.[19] This is in agreement with our findings also which showed good correlation between PAP and pulmonary function tests.

In our study, most patients presented during acute exacerbation of the disease and with PAP $>25 \mathrm{mmHg}$. Kessler et al. showed that the presence of a mean PAP $>2.4 \mathrm{kPa}(18$ $\mathrm{mmHg}$ ) was one of the best predictors of an increased risk of hospitalization for acute exacerbation. ${ }^{[5]}$ A study by Forfia et al. proved that the degree of TAPSE is a useful echo derived measure of RV function with prognostic significance in PAH.
It also powerfully reflects RV function and prognosis with in PAH.[20] A similar conclusion was drawn from a study by López-Candales A et al. and Hilde JM et al.[21,22] It was proven that TAPSE correlated well with right ventricular function. Hence TAPSE was taken as a measure of RV dysfunction with a cut off value of $<17 \mathrm{~mm}$. This was reflected well in our study as well. This indicates a majority of pulmonary artery hypertensive patients having RV dysfunction with a mean value of TAPSE being $16.16 \pm 3.42 \mathrm{~mm}$ in our study. However, in study by Mohammed et al., TAPSE was normal in all patients.[11]

It was well established by Vonk-Noordegraaf A et al. that concentric RV hypertrophy can precede resting hypoxia in patients with stable COPD and was demonstrated at autopsy in $76 \%$ of patients.[23] Similar findings can be extrapolated to our study wherein the number of patients with RV hypertrophy, as is indicated by RV thickness of $>5 \mathrm{~mm}$, was more than number of patients having a dilated RV. In our study, 34 patients had RV thickness $>5 \mathrm{~mm}$ while 23 patients had RV dilation with RV size $>41 \mathrm{~mm}$. This may be due to disease progression affecting RV thickness first in chronic COPD patients. Mohammed et al. and Jatav et al. found a positive correlation between severity of COPD and RV size.[11, 12]. In our study, PA pressure and RV hypertrophy have been established as having a significant correlation with severity of COPD as measured by FEV1 readings by spirometry. RV contractility and dysfunction also had significance with severity of COPD in our study.

RV contractility and dysfunction in the aforementioned study did not have significance with severity of COPD as is shown in our study. In a study by Kolb TM et al., it was established that right heart dysfunction in COPD patients is characterized by mildly increased PA pressures, RV hypertrophy, and preserved RV contractility and cardiac output.[24] However, RV contractility and dysfunction in the aforementioned study did not have significance with severity of COPD as is shown in our study. The hemodynamic variability of the patients due to acute exacerbations may be a reason for this variation in the studies. Numerous studies have proved association of PAH with COPD. Sims MW et al. conducted a trial on COPD cases with PAH and evaluated correlations with RA size, RA pressure, RV size, RV pressure and RV function.[25] PAP showed increasing trend with lowering FEV1 values, a trend seen in our study as well with only 4 of moderate COPD patients having PAH whereas all patients in very severe COPD category had PAH. Although in that study, prevalence of $\mathrm{PAH}$ was established to be $23 \%$ compared to $68 \%$ in our study. In general, patients presenting with acute exacerbations will have hypoxemia status which could be a factor influencing PAH status in our study.

In a study done by Hilde JM et al., COPD cases were examined along with controls for right ventricular dysfunction and remodeling in COPD cases.[22] RA size remained statistically insignificant. However, RA pressures were increased with mean value of $8.99 \pm 3 \mathrm{mmHg}$. In our study too, 26 patients had RA pressure $9 \pm 4.29 \mathrm{mmHg}$, with majority of patients having a RA pressure increase also having PAH. However a statistical significance correlation between severity of COPD and RA size and pressure could not be established, which was similar to study by Mutlu et al.[4] However, in our study, we had a higher incidence of PAH with 
a majority of COPD patients having PAH (34 out of 50). Also, our study had a statistically significant correlation between RA pressure and COPD severity. As hemodynamic parameters are greatly influenced by acute exacerbations of COPD, our study has variations due to presence of majority of patients having acute exacerbations were assessed clinically. Similarly, findings of calculated RVP of $40 \pm 9 \mathrm{mmHg}$ were seen in the pulmonary hypertensive patients of the study by Hilde JM et al.[22] In our study too, calculated RVP was $37.04 \pm$ $16.48 \mathrm{mmHg}$ with a statistically significant correlation of calculated right ventricular pressure with severity of COPD. Similar findings of RV thickness and TAPSE were seen in study by Hilde JM et al. versus our study (mean RV thickness $6.42 \pm 3.28 \mathrm{~mm}$ vs $7.16 \pm 2.98 \mathrm{~mm}$; TAPSE, $17 \pm 4 \mathrm{~mm}$ vs $16.16 \pm 3.42 \mathrm{~mm}$ respectively). The slight variations in mean values can be attributed to acute exacerbations of the disease.

In a study done by Agrawal $A$, the profile of echocardiographic changes in cor pulmonale was studied.[26] It was found that all cases had RV dilation, RA dilation, RV hypertrophy while $72 \%$ patients had RV dysfunction. It is difficult to extrapolate these findings to our study as we have not taken clinical findings of cor-pulmonale per se. It can be interpreted that patients having one feature of cor-pulmonale usually had all other right heart parameters deranged as well. As in, out of the 34 patients having PAH, all had RA and RV dilation, RV hypertrophy as well as RV dysfunction as shown by TAPSE. The same is true vice versa. In a study by Krishnan DR et al., a statistically significant correlation was seen between severity of COPD and the PAP and right ventricular systolic dysfunction.[27] Similar findings were found in our study with a statistically significant correlation between the above-mentioned parameters, along with other parameters of right heart. Similar findings of RA and RV dilation, RV hypertrophy as well as RV dysfunction as shown by TAPSE were proven to be statistically significant in a study by Gupta NK et al.[28]. The present study may have multiple clinical implications. The echocardiographic features of RV dysfunction and PAH may not be otherwise detected by routine investigations. ECHO in addition provides a simple tool to assess right heart function in long standing COPD cases to better prognosticate and manage them. It may be a sort of warning sign for further lifestyle management in such patients.

There were some limitations to our study. Our study was a single-center hospital-based study. Thus its results cannot be extrapolated to study the prevalence of COPD in the general population. Small sample size also limited our study.

\section{CONCLUSIONS}

Echocardiography is a great tool to evaluate right heart parameters in COPD. Significant prevalence of right heart dysfunction is seen in COPD and hence, COPD patients should be subject to right heart evaluation by echocardiography so that treatment modalities can be modified to reduce morbidity and mortality. Since statistically significant association is found between COPD severity and right heart parameters like RA pressure, RV size, RV pressure, PAH, RV thickness and TAPSE, echocardiography should be done at regular intervals, and especially during worsening of disease or acute exacerbations, so that treatment can be altered and modified with respect to hemodynamic parameters of the right heart for better patient care.

\section{REFERENCES}

[1] Medical Research Council. Definition and classification of chronic bronchitis for clinical and epidemiological purposes. A report to the medical research council by their committee on the aetiology of chronic bronchitis. Lancet 1965;285(7389):775-9.

[2] Doll R, Peto R, Wheatley $\mathrm{K}$, et al. Mortality in relation to smoking: 40 years observations on male British doctors. Br Med J 1994;309(6959):901-11.

[3] Borrows B, Knudson RJ, Cline MG, et al. Quantitative relationships between the cigarette smoking and ventilatory function. Am Rev Respir Dis 1977;115(2):195-205.

[4] Mutlu P, Aksit E, Mirici NA. Echocardiographic evaluation of right and left heart findings according to the severity of the disease in stable chronic obstructive pulmonary disease. Biomed Res 2018;29(11):2385-90.

[5] Kessler R, Faller M, Fourgaut G, et al. Predictive factors of hospitalization for acute exacerbation in a series of 64 patients with chronic obstructive pulmonary disease. Am J Resp Crit Care Med 1999;159(1):158-64.

[6] Weitzenblum E, Sautegeau A, Ehrhart M, et al. Long term course of pulmonary arterial pressure in chronic obstructive pulmonary disease. Am Rev Respir Dis 1984;130(6):993-8.

[7] Weitzenblum E, Hirth C, Ducolone A, et al. Prognostic value of pulmonary artery pressure in chronic obstructive pulmonary disease. Thorax 1981;36(10):752-8.

[8] Scharf SM, Iqbal M, Keller C, et al. Hemodynamic characterization of patients with severe emphysema. Am J Respir Crit Care Med 2002;166(3):314-22.

[9] Chaouat A, Schott R, Kessler R, et al. Severe pulmonary hypertension in chronic obstructive pulmonary disease. Am J Respir Crit Care Med 2003;167:A696.

[10] Abraham AS, Cole RB, Green ID, et al. Factors contributing to the reversible pulmonary hypertension of patients with acute respiratory failure studied by the serial observations during recovery. Circ Res 1969;24(1):51-60.

[11] Mohammed YM, ElShahid GS, Osman NM, et al. Cardiac evaluation of patients with chronic obstructive pulmonary disease using echocardiography. The Egypt J Bronchol 2019;13(1):12-6.

[12] Jatav VS, Meena SR, Jelia S, et al. Echocardiographic findings in chronic obstructive pulmonary disease and correlation of right ventricular dysfunction with disease severity. IJAM 2017;4(2):476-80.

[13] Moustapha A, Kaushik V, Diaz S, et al. Echocardiographic evaluation of left ventricular diastolic function in patients with chronic pulmonary hypertension. Cardiology 2001;95(2):96-100. 
[14] Schena M, Clini E, Errera D, et al. Echodoppler evaluation of lest ventricular impairment in chronic cor pulmonale. Chest 1996;109(6):1446-51.

[15] Trivedi HS, Joshi MN, Gamade AR. Echocardiography and pulmonary artery pressure. Correlation in chronic obstructive pulmonary disease. J Postgrad Med 1992;38(1):24-6.

[16] Watanabe T, Katsume E, Matsukubo H, et al. Estimation of right ventricular pressures with two dimensional echocardiography. Am J Cardiol 1982;49(8):1946-53.

[17] Walsh R, Fang J, Fuster V, et al. Hurst's the Heart Manual of Cardiology. 13 $3^{\text {th }}$ edn. McGraw-Hill Publishing 2012.

[18] Danchin N, Cornette A, Henriquez A, et al. Two dimensional echocardiographic assessment of the right ventricle in patients with chronic obstructive lung disease. Chest 1987;92(2):229-33.

[19] Higham MA, Dawson D, Joshi J, et al. Utility of echocardiography in assessment of pulmonary hypertension secondary to COPD. Eur Resp J 2001;17(3):350-5.

[20] Forfia PR, Fisher MR, Mathai SC, et al. Tricuspid annular displacement predicts survival in pulmonary hypertension. Am J Resp Crit Care Med 2006;174(9):1034-41.

[21] López-Candales A, Rajagopalan N, Saxena N, et al. Right ventricular systolic function is not the sole determinant of tricuspid annular motion. Am J Cardiol 2006;98(7):973-7.
[22] Hilde JM, Skjørten I, Grøtta OJ, et al. Right ventricular dysfunction and remodeling in chronic obstructive pulmonary dis- ease without pulmonary hypertension. J Am Coll Cardiol 2013;62(12):1103-11.

[23] Vonk-Noordegraaf A, Marcus JT, Holverda S, et al. Early changes of cardiac structure and function in COPD patients with mild hypoxemia. Chest 2005;127(6):1898903.

[24] Kolb TM, Hassoun PM. Right ventricular dysfunction in chronic lung disease. Cardiol Clin 2012;30(2):243-56.

[25] Sims MW, Margolis DJ, Localio AR, et al. Impact of pulmonary artery pressure on exercise function in severe COPD. Chest 2009;136(2):412-9.

[26] Agrawal A. Profile of echocardiographic changes in COR pulmonale. Int J Contemp Medical Res 2017;4(12):15-8.

[27] Krishnan DR, Srihari B. A study on the severity of right ventricular dysfunction in correlation with the severity of lung dysfunction in chronic obstructive pulmonary disease patients-COPD. Am J Sci Med Res 2015;1(1):1129.

[28] Gupta NK, Agarwal RK, Srivastav AB, et al. Echocardiographic evaluation of heart in chronic obstructive pulmonary disease patient and its corelation with the severity of disease. Lung India 2011;28(2):105-9. 